\title{
A Numerical Framework for Operational Solar Wind Prediction*)
}

\author{
Ljubomir NIKOLIĆ, Larisa TRICHTCHENKO and David BOTELER \\ Geomagnetic Laboratory, Natural Resources Canada, 7 Observatory Crescent, Ottawa, ON, K1A 0Y3, Canada
}

(Received 10 December 2013 / Accepted 17 February 2014)

\begin{abstract}
We report on the development of numerical components for an operational solar wind prediction framework. In particular, we discuss the coronal magnetic field component based on the potential field source surface and Schatten current sheet models, and the solar wind speed component which uses the empirical Wang-Sheeley-Arge relation between solar wind speed and open magnetic field lines. Using synoptic maps of photospheric fields in a stand-alone operational mode, the components provide the configuration of the global coronal magnetic field and prediction of the solar wind speed at 1AU. A good agreement between numerical prediction and solar wind speed observations has been found.
\end{abstract}

(c) 2014 The Japan Society of Plasma Science and Nuclear Fusion Research

Keywords: space weather, operational forecast, numerical modelling, solar wind, coronal holes, coronal field

DOI: $10.1585 /$ pfr.9.3406099

\section{Introduction}

Advances in technology in recent decades have been accompanied by increasing vulnerability to phenomena that can disrupt their safe operation. Simultaneously, modern society's growing dependence on these technological assets makes it even more important to provide forecasts of potentially damaging events. Space weather (SW) represents one of the natural hazards that in recent years is gaining prominence as a risk to human well-being. The fact that SW can have a major impact on technology has prompted establishment of research and operational strategies to understand and predict SW [1,2].

The term "Space Weather" refers to changes in the space environment and geomagnetic storms resulting from eruptions on the Sun. There is a growing interest in SW services from a wide range of stakeholders including: government agencies, power-grid companies, aviation, pipeline operators and global navigation satellite system users. Canada, with its high latitude location in the vicinity of the auroral zone is particularly vulnerable to the SW effects. The Canadian SW Forecast Center (CSWFC), Natural Resources Canada, monitors solar activity and parameters in the Sun-Earth system and provides information about SW conditions [3].

There is general agreement in the SW research and operational communities that more efforts should be put into the development of advanced SW prediction models. Most of the prediction models currently used in SW operations are still heavily based on empirical and semiempirical models. Despite great advances in large-scale simulations of space plasmas, in particular MHD, implementation of these techniques into operational SW predic-

author'se-mail: ljubomir.nikolic@nrcan-rncan.gc.ca

*) This article is based on the presentation at the 23rd International Toki Conference (ITC23). tions lags the research achievements. Just recently, the first 3D MHD SW operational code that provides 1-4 day advanced warning of Coronal Mass Ejection (CME) arrivals, has been implemented at the National Oceanic and Atmospheric Administration (US) [4,5].

CSWFC is developing advanced operational numerical models to predict changes in solar wind parameters close to the Earth. Our modular numerical simulation framework for the propagation of solar disturbances through interplanetary space, encompasses coronal magnetic field and solar wind speed components. These can provide initial parameters for an interplanetary 3D MHD simulation code. However, the coronal magnetic field and solar wind speed modules can also operate in a stand-alone configuration providing useful information to SW forecasters; including prediction of changes in the solar wind speed due to High Speed (HS) streams from coronal holes. Here, we report on the development of these components. In section 2 we briefly describe the numerical models and we discuss the results in section 3. A summary of the results and plans for future work are given in section 4 .

\section{Numerical Models}

\subsection{Coronal magnetic field}

To derive global magnetic field $\boldsymbol{B}$ of the solar corona from photospheric field observations, we use a Potential Field Source Surface (PFSS) model [6,7]. This model assumes existence of a fixed spherical "source surface" where the coronal magnetic field is purely radial. A radius of $r=R_{\mathrm{S}}=2.5 R_{0}$ for the source surface, where $R_{0}$ is the radius of the Sun, is widely accepted for the modelling and is used here. Although the fixed spherical surface assumption, together with neglecting coronal currents, poses a shortcoming of the PFSS model, the solution using MHD approach does not offer significant advantages. It 
has been found that PFSS and MHD models give similar global topology of the coronal magnetic field [8].

The PFSS solution for the coronal magnetic field is based on an assumption that there are no currents in the region $R_{0} \leq r \leq R_{\mathrm{S}}$, which means that the magnetic field can be expressed as the gradient of a scalar potential $\Psi$

$$
\nabla \times \boldsymbol{B}=0 \Rightarrow \boldsymbol{B}=-\nabla \Psi,
$$

which with the divergenceless property of the magnetic field $\nabla \cdot \boldsymbol{B}=0$, gives the Laplace equation for the potential

$$
\nabla^{2} \Psi=0
$$

Using the separation of variables $\Psi(r, \theta, \phi)=$ $R(r) \Theta(\theta) \Phi(\phi)$ in spherical coordinates, where $\theta \in[0, \pi]$ and $\phi \in[0,2 \pi]$, and assuming that at the source surface $r=R_{\mathrm{S}}$ the magnetic field is radial, i.e. $\Psi\left(R_{\mathrm{s}}, \theta, \phi\right)=$ const., we can express the solution of (2) in the region $R_{0} \leq r \leq R_{\mathrm{S}}$ in terms of spherical harmonics as

$$
\begin{aligned}
\Psi & =\sum_{n=1}^{\infty} \sum_{m=0}^{n} P_{n}^{m}(\cos \theta)\left(g_{n m} \cos m \phi+h_{n m} \sin m \phi\right) \\
& \times\left[R_{0}\left(\frac{R_{0}}{r}\right)^{n+1}-R_{\mathrm{S}}\left(\frac{R_{0}}{R_{\mathrm{S}}}\right)^{n+2}\left(\frac{r}{R_{\mathrm{S}}}\right)^{n}\right],
\end{aligned}
$$

where $P_{n}^{m}(\cos \theta)$ represent the associated Legendre polynomials. Using Eqs. 1 and 3 one can obtain the magnetic field components which, using orthogonality of Legendre polynomials, can be expressed as a function of the radial component of the photospheric magnetic field $B_{r}\left(R_{0}, \theta, \phi\right)$. Since this photospheric field can be derived from solar magnetograms, one can obtain realistic coronal magnetic field in the region $R_{0} \leq r \leq R_{\mathrm{s}}$. Moreover, using the Schatten current sheet model [9] it is possible to extend the coronal magnetic field beyond the source surface $R_{\mathrm{s}}$.

\subsection{Solar wind}

We employ the Wang-Sheeley-Arge (WSA) model to correlate solar wind speed $V_{\mathrm{sw}}$ with open magnetic field lines calculated using the PFSS coronal field model. In the WSA model, $V_{\text {sw }}$ depends on the flux tube expansion factor

$$
f_{\mathrm{s}}=\frac{\left|\boldsymbol{B}\left(R_{0}\right)\right|}{\left|\boldsymbol{B}\left(R_{\mathrm{s}}\right)\right|} \frac{R_{0}^{2}}{R_{\mathrm{s}}^{2}},
$$

and the angular separation between an open magnetic field line foot point and the coronal hole boundary $\theta_{\mathrm{b}}$ at the photosphere [10,11]. A general form of the relation between $V_{\mathrm{sw}}, f_{\mathrm{s}}$ and $\theta_{\mathrm{b}}$, can be expressed as [12],

$$
V_{\mathrm{sw}}=a_{1}+\frac{a_{2}}{\left(1+f_{\mathrm{s}}\right)^{a_{3}}}\left[a_{4}-a_{5} \exp \left\{-\left(\frac{\theta_{\mathrm{b}}}{a_{6}}\right)^{a_{7}}\right\}\right]^{a_{8}},
$$

where $a_{1}-a_{8}$ are empirical numerical coefficients. These coefficients are tunable parameters that depend on the magnetogram source used to derive the coronal magnetic field and calculation parameters. (a) $\mathrm{B}_{\mathrm{r}}[\mathrm{G}]$ GONG Date: 2012-02-23 Time: 14:04

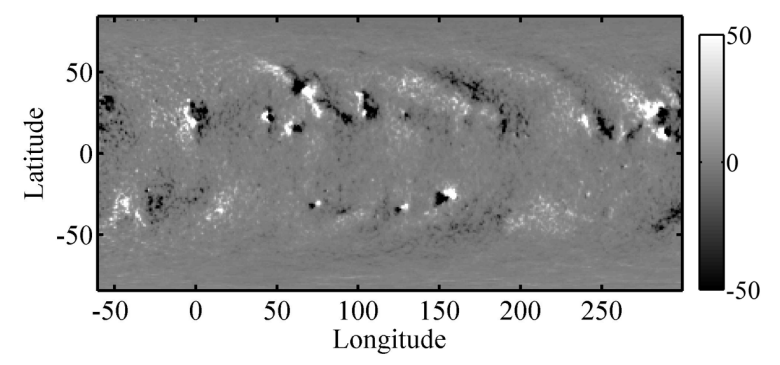

(b)

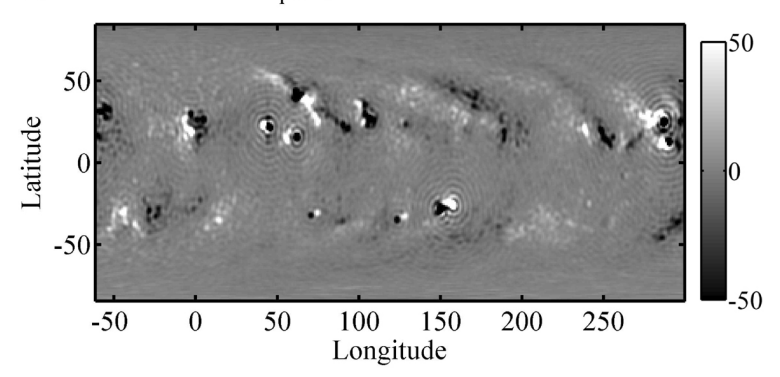

Fig. 1 Photospheric radial component of the magnetic field $B_{r}\left(R_{0}, \theta, \phi\right)$ from (a) the GONG synoptic magnetogram and (b) PFSS model with $n_{\max }=120$. The longitude $0^{\circ}$ corresponds to the central meridian on February 23, 2012 (14:04 UTC). The field scale is saturated at \pm 50 Gauss.

\section{Results and Discussion}

We have developed an operational numerical framework which consists of a number of Fortran and IDL modules. The framework runs eight times a day and uses hourly-updated Global Oscillation Network Group (GONG) magnetograms [13] and real time Advanced Composition Explorer (ACE) satellite solar wind measurements [14].

The standard GONG synoptic magnetograms on the $180 \times 360 \sin (\theta)-\phi$ grid are processed and serve as the input into the coronal magnetic field module. Here $\theta$ denotes the latitude and $\phi$ represents the longitude. In Fig. 1 (a), we show a classic GONG synoptic magnetogram. In order to better represent the details, the magnetic field scale is saturated in Fig. 1 at \pm 50 Gauss. The longitude $0^{\circ}$ corresponds to the central meridian on February 23, 2012 (14:04 UTC). The region on the left side of the central meridian will cross the meridian in coming days after 23rd. Using $B_{r}\left(R_{0}\right)$ from this synoptic map and PFSS numerical procedure described in the previous section the global magnetic field is obtained. Since the quality of the coronal field solution depends on the number of harmonics used in calculations (see Eq.3), we tested the agreement between the original magnetogram and the radial component of the magnetic field derived by the PFSS model in the limit $r=R_{0}$ using different number of harmonics. For a low number of harmonics, the fine details are often blurredout and a series of coarse ring-like structures appear in the derived photospheric field. An increase in the har- 
(a)

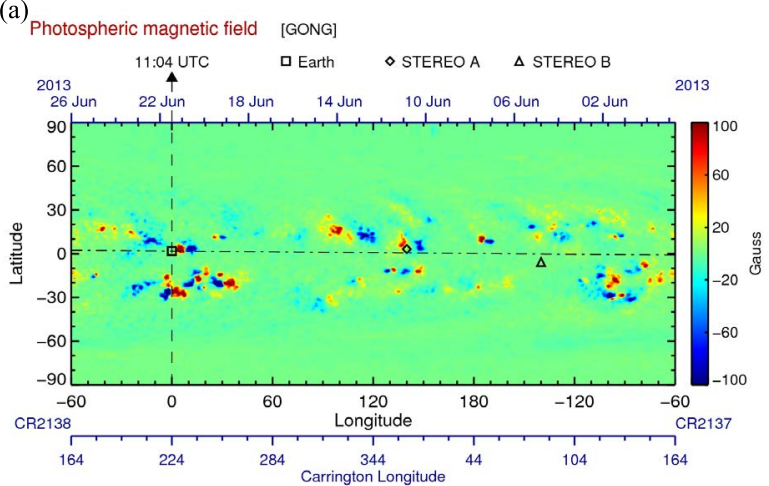

(b)

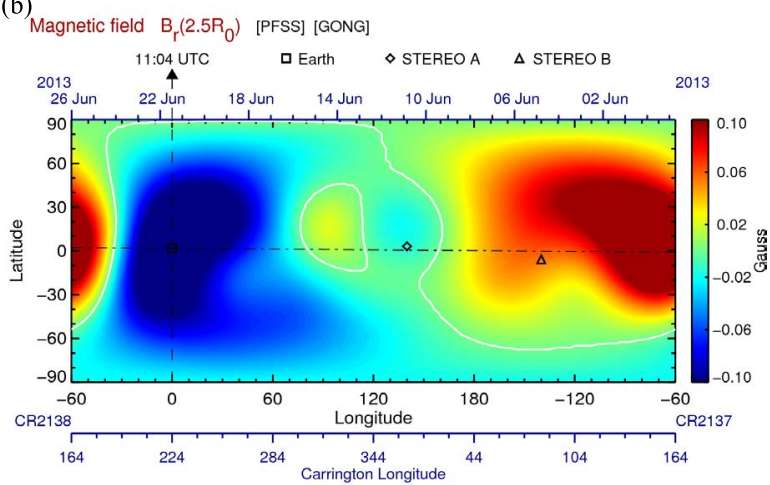

(c)

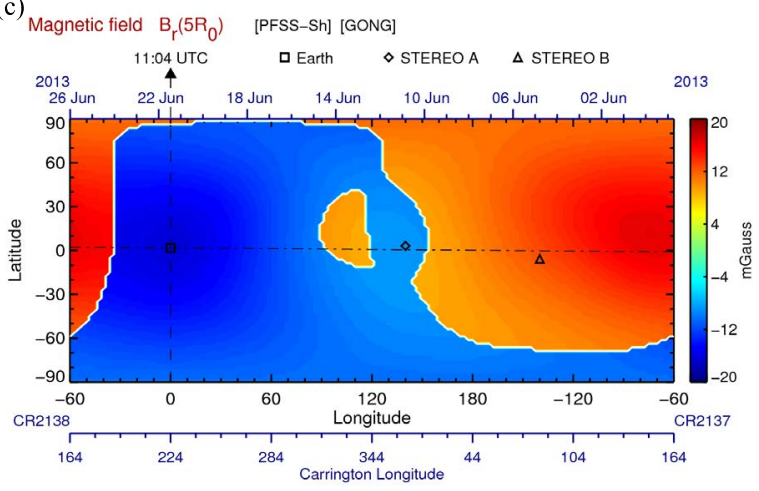

(d)

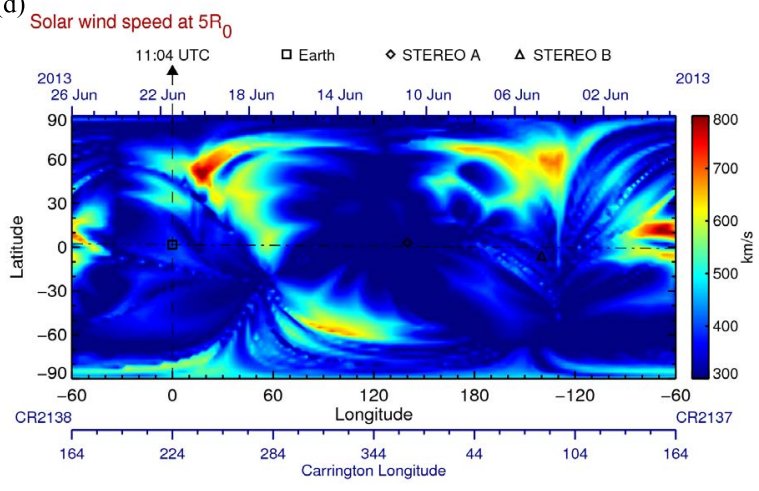

Fig. 2 (a) Photospheric magnetic field $B_{r}\left(R_{0}, \theta, \phi\right)$, (b) magnetic field at the source surface $B_{r}\left(2.5 R_{0}, \theta, \phi\right)$ from the PFSS model, (c) magnetic field $B_{r}\left(5 R_{0}, \theta, \phi\right)$ from the Schatten model, and (d) the solar wind speed $V_{\mathrm{sw}}\left(5 R_{0}, \theta, \phi\right)$ from the WSA model. The magnetogram used in the code is from June 21, 2013 (11:04 UTC). Dash-dash line represent the central meridian and dash-dot line the sub-earth positions. The scales are saturated at (a) \pm 100 Gauss, (b) \pm 0.1 Gauss, (c) $\pm 20 \mathrm{mGauss}$ and (d) 300 and $800 \mathrm{~km} / \mathrm{s}$. monics number improves the field reproducibility. In our standard calculations we use $n_{\max }=120\left(m \in\left[0, n_{\max }\right]\right)$. In Fig. 1 (b) we show the PFSS-derived photospheric field with $n_{\max }=120$. Although the small scale ring-like structures are still visible in Fig. 1 (b) there is a good agreement between observed and derived photospheric fields.

The real time visualization of active regions and derived coronal magnetic field provide $\mathrm{SW}$ forecasters with a source of information to draw conclusions about solar phenomena. In Fig. 2 (a), we show one of the typical outputs for the photospheric field showing a map of active regions and sub-earth positions (dash-dot line). Together with views of photospheric fields from STEREO A and B satellites (not shown), the images help to provide information about possible sources of solar flares and CMEs. Furthermore, coronal magnetic field images, such as Figs. 2 (b) and 2 (c), provide information about sector boundaries and open magnetic field polarity.

The results of the coronal magnetic field model are used to obtain the solar wind speed. We trace field lines using a Runge-Kutta method to find the corresponding foot point $\boldsymbol{B}\left(R_{0}\right)$ of an open magnetic field line $\boldsymbol{B}\left(R_{\mathrm{S}}\right)$, and to derive the flux tube expansion factor $f_{\mathrm{s}}$ and separation between open field foot point and its nearest coronal hole boundary $\theta_{\mathrm{b}}$. In our calculations we typically use the resolution of $2.5^{\circ} \times 2.5^{\circ}$. Using derived $f_{\mathrm{s}}$ and $\theta_{\mathrm{b}}$ we assign the solar wind speed to the open magnetic field lines (see Eq.5). For the empirical coefficients in Eq. 5 we use $a_{1}=250, a_{2}=875, a_{3}=1 / 4.5, a_{4}=1, a_{5}=0.8, a_{6}=2.6$, $a_{7}=1.25$ and $a_{8}=2.5$. An example of the solar wind speed map at $5 R_{0}$ obtained using this procedure is shown in Fig. 2 (d).

It is known that the coronal holes, dark regions seen in extreme-ultraviolet and X-ray images of the solar disc, are sources of HS solar wind streams and are associated with open magnetic field lines. In Fig. 3 (top) we show an example of modelled coronal holes for May 30, 2013. In this figure they are represented by regions that contain open magnetic field lines with $\left|\boldsymbol{B}\left(1.4 R_{0}\right)\right| /\left|\boldsymbol{B}\left(1.02 R_{0}\right)\right|>0.2$ at $r=1.4 R_{0}$. The modelled and observed coronal holes show a good agreement. To prove this, in Fig. 3 (bottom) we show observations of the central coronal hole by the Solar Dynamics Observatory (SDO) satellite.

The results presented in this section are from a standalone configuration, where the coronal magnetic field and solar wind numerical modules are decoupled from the MHD Sun-to-Earth interplanetary code. In this configuration, a simple kinematic model is used to propagate solar wind streams from the sub-earth positions at $5 R_{0}$ (from the dash-dot line in Fig. 2(d)) to the Earth. The prediction of the solar wind speed in many cases shows a good agreement with the observations. In Fig. 4, we compare predicted speed and the ACE satellite measurements for February 2007 (top) and June 2013 (bottom). As we can see a good match is obtained in February 2007 and in June $1-22,2013$. Note that the increase in the solar wind speed 

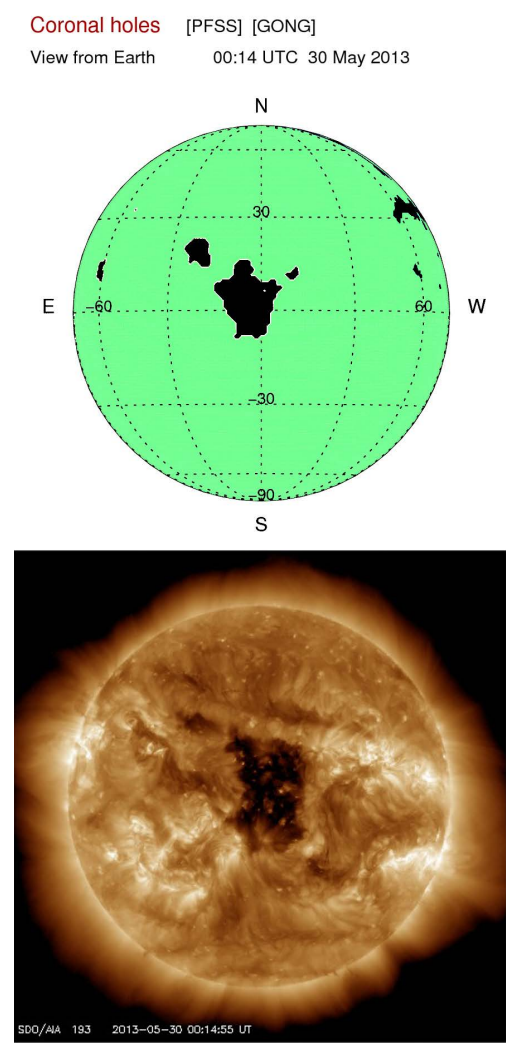

Fig. 3 Dark regions on the solar disc represent modelled (top) and observed coronal holes (SDO/AIA 193) (bottom) for May 30, 2013 (00:14 UTC). The central coronal hole is the source of HS solar wind streams shown in the Fig. 2(d) for May 30 - June 1, 2013.
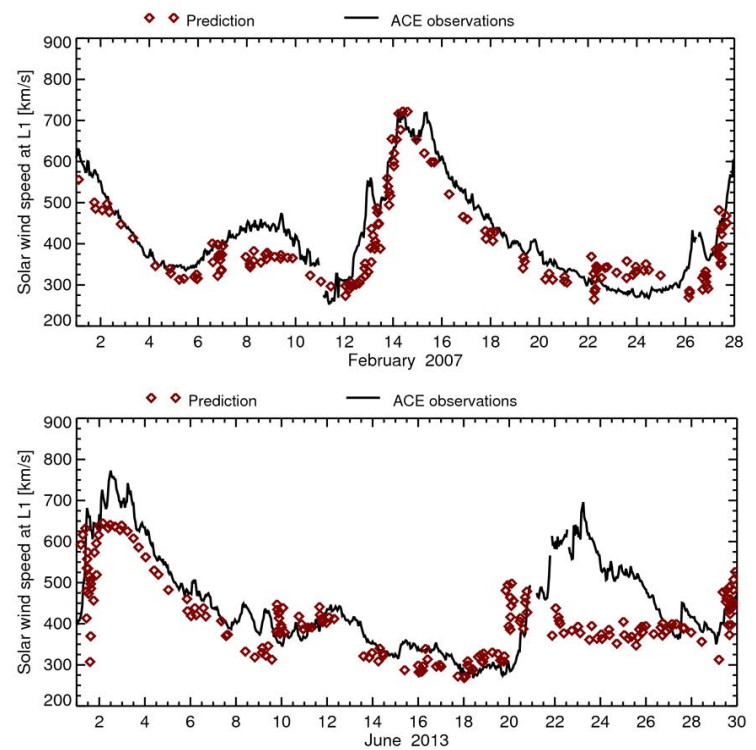

Fig. 4 Solar wind speed close to the Earth (L1 point) from the prediction model (red symbols) and ACE satellite measurements (black line) for February 2007 (top) and June 2013 (bottom). In the bottom figure, the strong enhancement in the solar wind speed for June 1-5 is due to HS streams from the coronal hole shown in Fig. 3. between June 1 and June 5, 2013, corresponds to the HS wind region shown in Fig. 2 (d) between May 30 and June 1. The source of this HS wind is the central coronal hole shown in Fig. 3.

While disagreements between predictions and observations of the solar wind speed (for example June 22-26, 2013, in Fig. 4) can be usually attributed to CMEs (which are not part of this prediction model configuration), there are cases that indicate that the disagreements are also due to non-accurate modelling of the solar wind sources. These disagreements challenge the simplified relation between $V_{\mathrm{sw}}, f_{\mathrm{s}}$ and $\theta_{\mathrm{b}}$ (see Eq. 5). In our future work we plan to address this issue and to re-examine the relation between coronal magnetic fields and solar wind properties.

\section{Summary}

We present work on the development of a numerical framework that aims to provide prediction of disturbances in solar wind parameters. Several components have been developed, including coronal field and solar wind speed modules. These can be used in a stand-alone configuration which offers fast satisfactory prediction 1-5 day in advance of changes in the solar wind speed due to the streams from coronal holes. In the fully developed framework, these components will be coupled with a CME module and our newly developed 3D MHD adaptive mesh refinement code to simulate Sun-to-Earth propagation of CMEs in the background solar wind.

\section{Acknowledgment}

This work utilizes data obtained by the Global Oscillation Network Group (GONG) Program, managed by the National Solar Observatory, which is operated by AURA, Inc. under a cooperative agreement with the National Science Foundation. The data were acquired by instruments operated by the Big Bear Solar Observatory, High Altitude Observatory, Learmonth Solar Observatory, Udaipur Solar Observatory, Instituto de Astrofísica de Canarias, and Cerro Tololo Interamerican Observatory. This work was performed as part of Natural Resources Canada's Public Safety Geoscience program with additional support from the Canadian Space Agency.

[1] H. Koskinen and T. Pulkkinen, "State of the art space weather modelling and proposed ESA strategy," SPEEWP310-TN (1998).

[2] National Space Weather Program Implementation Plan, FCM-P31-2000, US (2002).

[3] Space Weather Canada, Natural Resources Canada. http:// www.spaceweather.ca

[4] A. Taktakishvili et al., Space Weather 7, S03004 (2011).

[5] V.J. Pizzo et al., Space Weather 9, S03004 (2011).

[6] M.D. Altschuler and G. Newkirk, Sol. Phys. 9, 131 (1969).

[7] M.D. Altschuler et al., Sol. Phys. 51, 345 (1977).

[8] P. Riley et al., Astrophys. J. 653, 1510 (2006).

[9] K.H. Schatten, Cosmic Electrodynamics 2, 232 (1971). 
[10] C.N. Arge and V.J. Pizzo, J. Geophys. Res. 105, 465 (2000).

[11] C.N. Arge et al., Solar Wind Ten, Conference Proceedings 679, 190 (2003).

[12] P. MacNeice, Space Weather 7, S12002 (2009).
[13] National Solar Observatory, GONG. http://gong.nso.edu/

[14] Advanced Composition Explorer (ACE). http://www.srl. caltech.edu/ACE/ 